\title{
Using Interactive Screen Experiments as Pre-Laboratory Tasks to Enhance Student Learning
}

\section{S.J. Nolan, ${ }^{1,2}$ M. Szablewski, ${ }^{1}$ T.J.L. McComb ${ }^{1}$ \& I.G. Hughes ${ }^{1}$}

'Department of Physics, Durham University, South Road, Durham DH1 3LE, UK

${ }^{2}$ Foundation Centre, Durham University, Leazes Road, Durham DH1 1TA, UK

\section{Corresponding author:}

S.J. Nolan, Foundation Centre, Durham University, Leazes Road, Durham DH1 1TA, UK

Email: s.j.nolan@durham.ac.uk

\begin{abstract}
The teaching of first year undergraduate practical physics is currently faced with a difficult problem: the disparity in the level of practical physics many university entrant students have encountered prior to their arrival. Those with little practical physics experience enter the laboratory for the first time with a great deal of anxiety, which represents a barrier to their learning. This anxiety is magnified when their fellow students, some of whom have significant practical laboratory experience in their recent educational background, deal easily with the same situation. At Durham University, Interactive Screen Experiments (ISEs) have been used to familiarise students with laboratory equipment as part of an assessed pre-laboratory task for the firstyear physics laboratory, after which they perform real experiments.
\end{abstract}

ISEs are photograph-based virtualizations of real experiments that allow students to interact with a representation of laboratory equipment prior to entering a laboratory class. These interactive sessions enable students to learn by performing experiments virtually, in an environment where mistakes can be made safely and in private. This article contextualises the use of ISEs within their short but significant history. We first discuss their production and deployment and then focus on results from an evaluation of student perceptions of the use of ISEs. In contextualising the ISEs within the framework of the conversational model proposed by Laurillard, their ability to help 
overcome barriers to learning will be compared with their capacity to deepen and enhance learning.

Keywords: Physics, laboratory learning, interactive screen experiments, learning transition

\section{Introduction}

Practical laboratory learning is an essential component to any physics degree. The personal and scientific skills acquired in these sessions will be a key resource on which physics graduates can draw for years to come. At Durham University we have noticed that the variation in the practical physics experience of incoming undergraduate students has increased dramatically in recent years. Although the reasons for this are not obvious, in both their studies Smithers \& Robinson (2007) have noted that $41 \%$ of schools surveyed that taught up to GCSE level had no specialist physics teachers and that the schools that did not offer significant practical experience tended to lack technical support for the subject.

At university level these disparities in previous practical experience from student to student are significant. In informal discussions with students it quickly became apparent that students from schools where practical science was prioritised had had regular weekly practical physics lessons, and were adept at handling many types of scientific equipment used in the lab (micrometers, oscilloscopes, etc). However, the majority had had very few practical physics lessons, and so had no (or very little) familiarity with the equipment. This inexperience presented a significant barrier to learning and led to increased student anxiety during laboratory sessions. Solutions to this problem were sought; the approach chosen utilises Interactive Screen Experiments to simulate laboratory conditions, so that students can familiarise themselves with the equipment online, as part of a summative task, before entering the laboratory and working with the equipment in person. This short article discusses the origins of this project and how it was developed and deployed. The work will be linked to underlying elearning pedagogical theory, such as the conversational framework for learning proposed by Laurillard (2002), and a discussion of both its successes and limitations in this regard will be presented. Throughout, links to the broader research in this small but growing field will be presented.

\section{Interactive Screen Experiments}

Interactive Screen Experiments (hereafter ISEs) are photograph-based virtualisations of laboratory equipment; Hatherly defines them as "a highly interactive movie of an experiment, filmed as that experiment was being performed" (Hatherly et al. 2009). Over the past decade they have evolved, following the early pioneering work of Theyssen et al. (2002), Bacon (2004) and Kirstein \& Nordmeier (2007). The use of ISEs in this project differs from earlier work since here the ISEs are primarily deployed as pre-laboratory tasks to aid in overcoming a disparity of practical physics experience. The benefit of performing a task connected with an experiment before even entering the laboratory is well known. In relation to chemistry, for example, Johnstone \& Al-Shuailib (2001) state:

'Pre-laboratory preparation is not just read your manual before you come to the laboratory. ... The kind of pre-laboratory work that is being recommended must be as carefully prepared as the laboratory manual itself. It can take many forms, but it must prepare the student to be an active participant in the laboratory.'

The eight ISEs developed as part of this project were designed to familiarise students with the equipment and techniques they would be using in their next laboratory session and range from the simple but essential (e.g. calipers, micrometers) to the more complex (e.g. spectrometers, oscilloscopes). A screenshot of a prism spectrometer is shown in Figure 1.

The ISEs were developed by summer students (themselves veterans of the first-year laboratory course) over two successive summers. These students' role was to prepare the equipment, take successive photographic images of the laboratory equipment in every possible state, and then develop (using Adobe Flash) an interactive simulation of the apparatus using the images. The authors provided key advice for developing the simulations into useful teaching tools. The ISEs are available as Open Educational Resources from our course webpage and a guide to the production of ISEs is available from the authors upon request ${ }^{2}$.

\section{Results}

Although a quantitative study of the effect on student marks of ISE use has not been possible, the authors are aware of a number of qualitative factors indicating the positive impact the ISEs had in the first-year laboratory; these are discussed below in terms of student and staff perceptions.

\section{Student perceptions}

Student perceptions of the effect of a new technology on their learning are often hard to judge. In the 2011 and 2012 end-of-module 


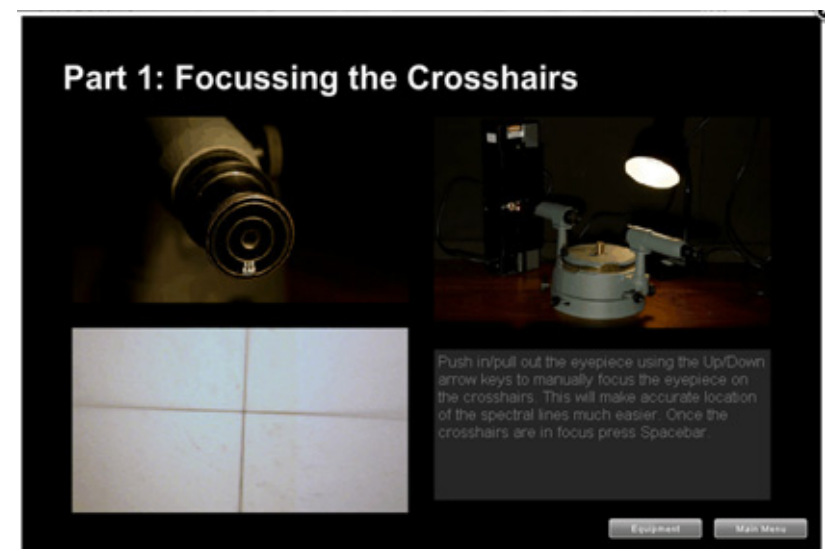

Figure 1 Screenshot of Prism Spectrometer ISE (available at: http://labs.physics.dur.ac.uk/level1/ISE/ISEs.php)

questionnaire, for example, students were asked their level of agreement with the statement:

"The interactive screen experiments (ISEs) provided as preparatory tasks for some of the Full Experiments helped my understanding of the experiment."

In both cases over $70 \%$ of the students agreed (or strongly agreed) with this statement. Informal student feedback was also positive. Students stated that they wished more experiments had ISE counterparts, and that they used the ISEs both prior to the lab, after the lab and when writing reports based around their work in the laboratory. Students are often almost completely task-orientated during a practical but appreciate the opportunity to access the experiments when writing up. ISEs used during project write-up allow students to quickly recall the session and to reflect more deeply on the scientific concepts involved.

\section{Staff perceptions}

Given that there are over 250 students taking this module, taught in groups of up to 16 , their teaching involves almost a dozen teaching staff. In informal discussions, the staff were generally positive about the use of ISEs as pre-laboratory tasks. Many commented that the amount of preparation time at the start of the laboratory session has decreased significantly, whilst others noted that student questions have changed from the procedural ("Where does this wire go?") to be more physicsbased ("which way do the electrons flow?") after introduction of the ISEs.

\section{Discussion}

In her seminal work, Rethinking University teaching, Laurillard proposed a framework for teaching based around a conversational approach between student and teacher. Laurillard studies different e-learning technologies within the context of this conversational framework, and examines how close each gets to capitalising on its strengths. In what follows, Laurillard's tenets for a good conversational framework are discussed in turn with reference to the ISEs developed here. Laurillard identifies a good conversational framework as one that:

Must operate a constructive iterative dialogue between teacher and student

Media such as ISEs must aim not only to simulate the experiment, but also to successfully simulate the dialogue a teacher and student would have in the laboratory. If introducing a new piece of apparatus (an oscilloscope, for example) in a laboratory, one might simply explain how it works and what the key buttons do. This is replicated in the ISE when students are given explanatory pictures and photographs. The next stage in laboratory teaching would be to set the student a task, and the ISE does not differ in this regard. Where the ISE falls short, of course, is in dealing with student questions as they arise, which any laboratory demonstrator would acknowledge is key to helping students learn. In ISEs the student is left with a choice of (a) randomly pressing buttons until the correct answer is found; (b) asking a friend; or (c) waiting until the next laboratory session to ask the demonstrator.

Must be discursive, adaptive, interactive, reflective and blended

The ISEs developed in this project were originally intended to be solely part of the preparatory task which students carry out before entering the lab. However, it was found that tutor-initiated discussion during the lab, focused around both the ISE and the equipment, allowed deeper learning to take place as the students compared the real world and the simulated ISE world. Furthermore, the ISEs are by their nature adaptive and interactive as they permit interaction between students and equipment virtually. Students then have time to reflect on their use of the ISE before being confronted with the 'real' version in their next laboratory session. 
Must operate at the level of descriptions of the topic

In regard to this criterion, ISEs clearly struggle. The aim of this project is to familiarise students with laboratory equipment prior to them using it in the lab. It is therefore intimately task-linked and although discussions with each other, and with their tutor, once in the lab will connect the use of the equipment to a larger physics-related aim (for example oscilloscopes are used to measure the speed of light in one experiment), if taken in isolation the ISE is clearly task-heavy and topic-light. As the laboratory itself focuses on the larger (physics-related) aim, if taken as part of a greater whole, the ISE is balanced in both topic and task.

Must also operate at the level of action within related tasks

In contrast to the above, the ISEs sit naturally within this criterion, as their aim is to introduce a piece of equipment and allow the students to engage with tasks which utilise the simulated equipment. This then builds directly into the laboratory session, since students can perform the same tasks and check their understanding.

\section{Conclusions and future work}

The development of Interactive Screen Experiments at Durham University's Physics Department has been discussed. Used as pre-laboratory tasks, ISEs were shown qualitatively to address the disparity of experience of practical physics between incoming first-year undergraduate students. Another potential use of ISEs will be explored in future work, where ISEs are being developed to help mature local students overcome barriers to learning presented by a lack of recent experience in the laboratory. In addition the use of ISEs to prepare international students for laboratory learning in the UK is being investigated. Some countries teach science with a significant bias to the theoretical over the experimental (Su et al. 1994 and Kind 1999) . Students from these countries may benefit from the use of ISEs pre-arrival to help them develop a working knowledge of laboratory equipment and aid them in developing their subject-specific language skills.

\section{Acknowledgements}

The authors thank Durham University for funding this work through E-Learning Development and Enhancing the Student Learning Experience awards. We also wish to thank our summer students Dan Maxwell and Tom Mitchell, who played a vital role in the development of our ISEs and who are now both physics Ph.D. students at Durham.

\section{References}

Bacon, R. (2004) Simulations for Physics and Astronomy. LTSN Physical Science News, 5, 10.

Hatherly, P.A., Jordan, S.E. and Cayless, A. (2009) Interactive screen experiments: innovative virtual laboratories for distance learners. European Journal of Physics, 30, 751-762.

Johnstone, A. and Al-Shuailib, A. (2001) Learning in the laboratory; some thoughts from the literature. University Chemistry Education, 5, 42.

Kind, P. (1999) TIMSS Performance Assessment-a cross national comparison of practical work, Practical Work in Science Education, Dordrecht: Kluwer Academic Publishers.

Kirstein, J. and Nordmeier, V. (2007) Multimedia representation of experiments in physics. European Journal of Physics, 28, S115.
Laurillard, D. (2002) Rethinking University Teaching: A

Framework for the Effective Use of Educational Technology, London: Routledge.

Smithers, A. and Robinson, P. (2007a) Physics in schools and universities: Bucking the trend. Buckingham: University of Buckingham, Centre for Education and Employment Research.

Smithers, A. and Robinson, P. (2007b) Physics in schools and universities: Supply and Retention of Teachers. Buckingham: University of Buckingham, Centre for Education and Employment Research.

Su, Z., Jilin, S. and Goldstein, S. (1994) Teaching and Learning Science in American and Chinese High Schools: A Comparative Study, Comparative Education, 30, 255.

Theyssen, H., Aufschnaiter, S.V. and Schumacher, D. (2002) Development and Evaluation in a Laboratory Course in Physics for Medical Students, Teaching and Learning in the Science Laboratory, Dordrecht: Kluwer Academic Publishers. 